\title{
As facilidades e contribuições da tecnologia point of care no ambiente hospitalar
}

\section{The facilities and contributions of point of care technology in the hospital environment}

Las instalaciones y aportaciones de la tecnología del punto de atención en el entorno hospitalario

Marlene Simões e Silva ORCID: https://orcid.org/0000-0002-1195-493X Universidade Federal de Minas Gerais, Brasil E-mail: marlene.simoes@ebserh.gov.br Pamela Nery do Lago ORCID: https://orcid.org/0000-0002-3421-1346 Universidade Federal de Minas Gerais, Brasil E-mail: pamela.lago@ebserh.gov.br

Vinícius Martins Machado

ORCID: https://orcid.org/0000-0002-6306-6379 Santa Casa de Misericórdia de Belo Horizonte, Brasil Universidade Federal de Minas Gerais, Brasil E-mail: machadoih@yahoo.com.br

Eliseu da Costa Campos

ORCID: https://orcid.org/0000-0002-1670-9626

Universidade Federal do Triângulo Mineiro, Brasil E-mail: eliseu.da.costa@usp.br

Samanntha Lara da Silva Torres Anaisse

ORCID: https://orcid.org/0000-0002-8350-5607

Universidade Federal do Mato Grosso do Sul, Brasil

E-mail: samanntha.anaisse@ebserh.gov.br

Marta Luiza da Cruz

ORCID: https://orcid.org/0000-0002-8946-2644

Universidade Federal do Mato Grosso do Sul, Brasil E-mail: martaluiza_cruz@hotmail.com

Tatiana Alves Costa

ORCID: https://orcid.org/0000-0002-5059-0796

Universidade Federal do Mato Grosso do Sul, Brasil

E-mail: tatiana.costa@ebserh.gov.br

Adriana Simões Moreira Rocha

ORCID: https://orcid.org/0000-0001-5025-0073

Universidade Federal da Bahia, Brasil

E-mail: adrianasmrocha@gmail.com

Andréa Paula Dourado Vasconcelos

ORCID: https://orcid.org/0000-0002-3619-4754

Universidade Federal do Pernambuco, Brasil

E-mail: andreapdourado@hotmail.com

Lívia Sayonara de Sousa Nascimento ORCID: https://orcid.org/0000-0003-2552-3169

Universidade Federal da Paraíba, Brasil E-mail: livia.nascimento@ebserh.gov.br

Pedro Henrique Santos Oliveira

ORCID: https://orcid.org/0000-0002-4641-8117 Faculdade de Ciências Sociais Aplicadas, Brasil

E-mail: phs89oliveira@gmail.com

Eugênio Barros Bortoluzi

ORCID: https://orcid.org/0000-0001-9074-8749 Faculdade de Ciências Sociais Aplicadas, Brasil

E-mail: eugenio@bortoluzi.com.br

Renata Cavalcanti Cordeiro

ORCID: https://orcid.org/0000-0001-6395-4811

Faculdade de Ciências Sociais Aplicadas, Brasil

E-mail: renatacc@outlook.com

Amanda Araújo Brandão

ORCID: https://orcid.org/0000-0002-4800-193X

Faculdade de Ciências Sociais Aplicadas, Brasil

E-mail: amanda.araujo.brandao@maisunifacisa.com.br

Ítallo Bernardo Souto

ORCID: https://orcid.org/0000-0001-6564-8957 Faculdade de Ciências Sociais Aplicadas, Brasil E-mail: itallobernardo77@gmail.com 


\begin{abstract}
Resumo
O objetivo deste estudo foi destacar as vantagens e contribuições trazidas pelos exames point of care apontando os consensos sobre esta temática na atualidade. Foi realizada uma revisão de literatura, elaborada através de coleta de dados a partir de fontes secundárias, por meio de levantamento bibliográfico. A busca se deu por documentos primários, através da base de dados da Scielo, Biblioteca Virtual da Saúde (BVS) e PubMed. Como critério de inclusão delimitou-se o período de 2011 a 2021, aceitando-se publicações na íntegra ou somente seus resumos publicados. Foram aceitos somente artigos publicados nos idiomas português e inglês. Como descritores foram utilizados: point of care, tecnologia, diagnóstico. Através do levantamento bibliográfico realizado foi possível concluir que a tecnologia point of care é sobremodo importante, até mesmo essencial para que se possa determinar o diagnóstico definitivo e confirmatório de uma série de condições patológicas e clínicas no cotidiano do atendimento hospitalar, especialmente. Esta tecnologia deve ser cada vez mais estimulada na prática, tendo em vista não somente o fato de que permite um diagnóstico precoce, mas também porque possibilita um melhor prognóstico para aqueles casos considerados mais graves, contribuindo para que vidas sejam salvas.
\end{abstract}

Palavras-chave: Point of care; Diagnóstico; Tecnologia.

\begin{abstract}
The aim of this study was to highlight the advantages and contributions brought by the point of care exams, pointing out the current consensus on this topic. A literature review was carried out, elaborated through data collection from secondary sources, through a bibliographic survey. The search for primary documents was carried out through the Scielo database, the Virtual Health Library (VHL) and PubMed. As inclusion criteria, the period from 2011 to 2021 was delimited, accepting full publications or only their published abstracts. Only articles published in Portuguese and English were accepted. The following descriptors were used: point of care, technology, diagnosis. Through the bibliographical survey carried out, it was possible to conclude that the point of care technology is extremely important, even essential for determining the definitive and confirmatory diagnosis of a series of pathological and clinical conditions in the daily routine of hospital care, especially. This technology should be increasingly encouraged in practice, considering not only the fact that it allows for an early diagnosis, but also because it provides a better prognosis for those cases considered more serious, contributing to the saving of lives.
\end{abstract}

Keywords: Point of care; Diagnosis; Technology.

\title{
Resumen
}

El objetivo de este estudio fue resaltar las ventajas y aportes que aportan los exámenes point of care, señalando el consenso actual sobre este tema. Se realizó una revisión de la literatura, elaborada a través de la recolección de datos de fuentes secundarias, mediante un relevamiento bibliográfico. La búsqueda de documentos primarios se realizó a través de la base de datos Scielo, la Biblioteca Virtual en Salud (BVS) y PubMed. Como criterio de inclusión se delimitó el período de 2011 a 2021, aceptando publicaciones completas o solo sus resúmenes publicados. Solo se aceptaron artículos publicados en portugués e inglés. Se utilizaron los siguientes descriptores: punto de atención, tecnología, diagnóstico. A través del relevamiento bibliográfico realizado, se pudo concluir que la tecnología del punto de atención es sumamente importante, incluso fundamental para determinar el diagnóstico definitivo y confirmatorio de una serie de condiciones patológicas y clínicas en la rutina diaria de la atención hospitalaria, especialmente. Esta tecnología debe ser impulsada cada vez más en la práctica, considerando no solo el hecho de que permite un diagnóstico precoz, sino también porque brinda un mejor pronóstico para aquellos casos considerados más graves, contribuyendo a salvar vidas.

Palabras clave: Punto de atención; Diagnóstico; Tecnología.

\section{Introdução}

O mercado de saúde está em constante transformação, envolvendo pesquisas científicas, equipamentos cada vez mais eficientes e autônomos, novos tratamentos, inovações em tecnologia da informação para criação de algoritmos preditivos de doenças crônicas e gestão populacional dos pacientes.

Cada vez mais recursos e dispositivos têm sido criados no intuito de contribuir de uma forma mais decisiva na facilitação e na melhoria da qualidade dos serviços prestados, especialmente no ambiente hospitalar, onde a excelência e a qualidade são sempre a prioridade para que se haja segurança do paciente.

A criação de dispositivos tecnológicos e ferramentas informatizadas pode representar uma importante contribuição também para a promoção de uma assistência mais humanizada e eficiente, agregando valor a todos os envolvidos e contribuindo de forma significativa na prestação de serviços de qualidade em um mercado altamente competitivo.

Assim, as ferramentas tecnológicas e sistematizadas a serviço das ciências da saúde devem ser analisadas e, diante da 
constatação de sua eficiência, devem ser colocadas em prática nas atividades rotineiras realizadas a nível hospitalar.

Um importante recurso desenvolvido nas últimas décadas foi o chamado teste point of care (POC), também conhecidos no Brasil como testes laboratoriais rápidos ou exames a beira leito, como a ultrassonografia, os quais têm como principal objetivo a realização de diagnósticos de forma mais rápida e eficiente (Dusse, Oliveira, Rios \& Marcolino, 2012). Neste contexto, apresentam inúmeras vantagens para o diagnóstico e acompanhamento de diversas doenças (Celer Biotecnologia, 2021).

Neste cenário, o objetivo deste estudo é destacar as vantagens e contribuições trazidas pelos testes point of care apontando os consensos da literatura sobre esta temática na atualidade.

\section{Metodologia}

Trata-se de um estudo de revisão bibliográfica, do tipo narrativa e de cunho descritivo qualitativo (Marconi \& Lakatos, 2017) elaborado através de coleta de dados realizada a partir de fontes secundárias, por meio de levantamento bibliográfico.

As etapas, que possibilitaram a operacionalização da revisão, foram iniciadas pela busca por documentos primários, através da base de dados da Scielo, Biblioteca Virtual da Saúde (BVS) e PubMed. Como descritores, foram utilizados: point of care, tecnologia, diagnóstico.

Como critério de inclusão delimitou-se o período de 2011 a 2021, aceitando-se publicações na íntegra ou somente seus resumos publicados, nos idiomas português e inglês.

Foram excluídas as literaturas com mais de 11 anos de publicação e os que não contribuíssem diretamente para pesquisa. Para seleção do material foram analisados e selecionados com base nos títulos e posteriormente nos resumos, visando analisar as vantagens e contribuições trazidas pelos testes point of care. Por fim, realizada a análise dos dados coletados para 0 desenvolvimento do mesmo e elaboração das considerações finais acerca do presente estudo, instituindo consonância com os objetivos fundamentados (Marconi \& Lakatos, 2017).

Aqui são apresentados os 20 estudos de maior relevância e que atenderam os critérios de elegibilidade.

\section{Resultados e Discussão}

A tecnologia POC vem para auxiliar na medicina diagnóstica, promovendo um verdadeiro salto tecnológico, principalmente quando pensamos em locais distantes e remotos, pois possibilita sobremaneira a realização de exames que por outros meios seriam mais onerosos, demorados e por vezes inacessíveis.

Para Celer Biotecnologia (2021, p. 1):

Esse mercado não para de crescer, sobretudo em países como o Brasil, com sua grande população e alta prevalência de doenças crônicas, que necessitam de monitoramento constante.

Em todo o mundo, as empresas do setor estão investindo todos os seus esforços para acelerar seus processos de inovação. Esse salto tecnológico que iremos presenciar poderá contribuir de forma relevante para aprimorar os processos de atenção à saúde.

Sobretudo em um momento em que o mundo passa por uma grave crise de saúde pública, devido à pandemia de COVID-19, essas inovações são mais do que bem-vindas.

Arienzo et al. (2019) afirmam que um diagnóstico rápido e preciso é essencial para garantir uma terapia oportuna e eficaz. No caso, por exemplo, dos testes para diagnóstico de infecção do trato urinário (ITU), juntamente com os métodos de referência baseados na cultura, vários testes POC para deteç̧ão precoce de ITUs foram desenvolvidos. Sendo, portanto, métodos que trazem agilidade para a escolha do tratamento adequado. 
A Organização Mundial da Saúde (OMS) recomenda o uso do teste diagnóstico POC de Cryptococcal Antigen Lateral Flow Assay (CRAG-LFA) pois:

O diagnóstico e o tratamento precoce da meningite criptocócica são essenciais para reduzir a mortalidade por doença criptocócica em pacientes com infecção pelo vírus da imunodeficiência humana (HIV). A recomendação do órgão é para que os países deem prioridade a ensaios de antígeno criptocócico de diagnóstico rápido, de preferência ensaios de fluxo lateral como o CRAG-LFA, para uso no líquor, soro, plasma ou sangue total (Brasil, 2021, p. 27).

Além das questões relacionadas com a agilidade do diagnóstico e início do tratamento, deve-se considerar de forma não menos importante, que se comparado aos exames convencionais, os testes POC tem um custo bem menor, sendo assim, possível realizar os testes em uma parcela maior da população.

Leal et al. (2013) assinalam a importância dos testes POC no período pré-operatório, principalmente quando trata-se de procedimentos de urgência, que requerem uma resposta rápida para uma decisão assertiva, visto que uma avaliação préoperatória adequada é fundamental para otimizar o respectivo procedimento para cada paciente, reduzindo riscos e custos.

Phillips et al. (2019) destacam que os profissionais de saúde que têm acesso aos testes POC estão cada vez mais solicitando o mesmo desempenho destes em comparação ao que esperam dos exames laboratoriais. Com a introdução do instrumento Cobas ${ }^{\circledR}$ Liat, testes de diagnóstico molecular altamente sensíveis podem ser realizados mais perto do paciente em configurações de POC.

Crocker et al. (2014) afirmaram que os testes laboratoriais POC oferecem tempo de resposta reduzido e pode promover melhor eficiência operacional. No estudo destes autores, após a implementação do POC, houve uma redução de $21 \%$ nos testes solicitados por paciente; uma redução de 89\% nas chamadas; $85 \%$ nos telefonemas de acompanhamento e $61 \%$ de diminuição nas revisitas dos pacientes. Concluiu-se que o POC pode melhorar significativamente os fluxos com redução de custos através de práticas aprimoradas.

Uma importante questão a respeito da implantação do POC refere-se à diminuição de custos em muitas regiões do país de difícil acesso, a exemplo do estudo desenvolvido por Noronha (2019) em que o autor avalia a implantação e utilização da tecnologia POC em serviços públicos de saúde visando mitigar problemas de ordem econômica, uma vez que os laboratórios públicos não oferecem o serviço diagnóstico 24 horas por dia, especialmente em regiões onde não existem hospitais com laboratórios integrados.

De acordo com Schiling (2014), no Canadá, o uso de testes POC durante a estabilização dos pacientes antes da transferência inter-hospitalar foi calculada, resultando em uma economia substancial de tempo. Ainda, nos EUA, a implementação de POC resultou em uma diminuição de $20 \%$ nas internações na unidade de dor torácica e consequentemente uma economia substancial. Em cirurgia cardiotorácica com alta utilização de componentes sanguíneos caros, a introdução da medição da coagulação por teste POC resultou em economia de 56\%. Na unidade de observação cardíaca, a introdução de troponina reduziu o tempo de permanência, taxas de admissão diminuídas e resultou em menos procedimentos dispendiosos, com uma economia de $25 \%$ nos custos por paciente.

Bollinger e Tanaka (2017) consideram que o teste de coagulação POC permite decisões clínicas rápidas em intervenções hematológicas e, quando usado em conjunto com um algoritmo de transfusão adequado, pode reduzir o uso de hemoderivados e complicações potencialmente associadas à transfusão de sangue

Schiling (2014) afirmou que o uso de sistemas POC permite a análise rápida de amostras de sangue especialmente em unidades de emergência, trazendo inúmeras vantagens. Neste estudo, analisou-se o potencial impacto econômico do uso do sistema iSTAT POC em uma unidade de emergência de um hospital universitário. Observou-se que o uso do POC resultou em economia direta por análise de US\$ 111,00 para cada paciente. O atraso médio entre os resultados do POC e os resultados 
laboratoriais foi de 48,5 $\pm 28,67$ minutos. Isso é equivalente a 32.333 horas de trabalho por ano. Isso contabiliza em termos de recursos humanos do setor de emergência um custo de US\$ 1,5 por paciente/minuto. O tempo economizado pelo POC se traduz em uma economia potencial de US\$ 72,75 por paciente. Baseado em 40.000 visitas de pacientes por ano no prontosocorro, US\$ 7.350.000 podem ser economizados pelo uso rigoroso do POC. Os autores concluíram que o uso do POC pode produzir um grande impacto econômico para a unidade de emergência se todas as economias potenciais forem realizadas. Considerando a economia potencial em tempos de espera, o POC também pode aumentar nos pacientes a segurança e satisfação, além de ajudar a diminuir a superlotação destas unidades hospitalares.

De acordo com Schiling (2014), na UTI neonatal, o teste POC demonstrou reduzir os custos por paciente em 8,3\% como resultado da redução de testes laboratoriais principais e menos transfusões. No pronto-socorro pediátrico, a introdução do teste POC para detecção de vírus sincicial respiratório humano na triagem de pacientes foi significativamente alterada, trazendo mais rapidez às decisões terapêuticas bem como aos procedimentos de isolamento para tais, na medida em que era possível liberar leitos durante os períodos de epidemia, com grandes consequências econômicas.

Aljadi et al. (2019) citaram que o teste de ativação de basófilos (BAT) baseado em citometria de fluxo é usado para o diagnóstico de resposta alérgica. No entanto, a citometria de fluxo é demorada, exigindo pessoal qualificado e processamento complicado, o que limitou seu uso na clínica. Os autores apresentaram um novo método de imunoafinidade BAT (miBAT) baseado em microfluidicação. Concluiu-se que este dispositivo é capaz de isolar basófilos diretamente do sangue total para ativação e detecção no chip. O novo método miBAT aguarda validação em populações maiores de pacientes para avaliar o desempenho no diagnóstico e monitoramento de pacientes.

Lusignan et al. (2019) destacaram que o teste POC para influenza promete fornecer informações em tempo real para influenciar a tomada de decisão clínica e melhorar os resultados do paciente. A Public Health England publicou um kit de ferramentas para auxiliar a implementação desses testes no Serviço Nacional de Saúde do Reino Unido. Os autores realizaram um estudo de viabilidade para avaliar a implementação da POC para influenza na atenção primária, como parte de uma rede de vigilância sentinela. Foi conduzido um estudo de métodos mistos para comparar as taxas de amostragem em práticas que usam o POC e as atuais práticas de swab de virologia que não usam o POC, e para entender os problemas e as barreiras à implementação do POC para influenza nos fluxos de trabalho da atenção primária. Foram recrutadas 6 práticas e a coleta de dados foi iniciada em 11 de março de 2019. Além disso, foram coletadas 312 amostras de swab no momento da submissão do protocolo, que era de 32,5\% (312/960) do tamanho esperado da amostra. Além disso, 68 amostras foram positivas para influenza, que era 20,1\% (68/338) do tamanho esperado da amostra. Concluiu-se que é de grande viabilidade a implementação do POC na atenção primária e as opiniões dos profissionais sobre o seu uso na atenção primária, incluindo seu impacto nos fluxos de trabalho.

Barcellona et al. (2017) afirmaram que as terapias anticoagulantes orais com os medicamentos anti-vitamina $\mathrm{K}$ (AVK), varfarina, acenocumarol e fenprocume, são empregadas na profilaxia antitrombótica primária e secundária em pacientes com tromboembolismo venoso, fibrilação atrial e válvulas mecânicas cardíacas. A telemedicina pode fornecer ajuda significativa no gerenciamento dessa terapia, permitindo que os pacientes realizem o teste em casa ou em qualquer outro lugar com um dispositivo portátil, ou seja, teste POC e enviem o resultado para a equipe responsável pelo seu atendimento, e então, os pacientes podem receber ajuste de dose. A eficácia desse tipo de manejo é igual ou superior ao monitoramento tradicional do AVK em termos de eventos hemorrágicos e trombóticos. A análise dos custos com um horizonte de 10 anos revela que tanto o autoteste quanto o autogerenciamento são testes baratos.

Nunes et al. (2016) citaram que a ultrassonografia à beira do leito (POCUS) - do inglês point of care ultrasonography - ocupa um papel propedêutico de crescente importância no diagnóstico clínico. Em geral, os clínicos têm demonstrado habilidade na obtenção de imagens que os permitem responderem questões simples, tipicamente binomiais. A integração 
dessas imagens ao histórico clínico e aos resultados do exame físico tem aprimorado o manejo dos pacientes por seus clínicos. Os médicos estão demonstrando interesse na POCUS, e algumas escolas de medicina, particularmente nos EUA, já introduziram a ultrassonografia em seus currículos de graduação. No Brasil, a Faculdade de Medicina da Universidade Federal de Juiz de Fora está desenvolvendo uma iniciativa pioneira que envolve a incorporação estruturada e horizontal da POCUS no currículo escolar.

Em um estudo realizado por Nunes et al. (2016) os autores buscaram apresentar os resultados do desenvolvimento de competências para o uso do ultrassom POCUS em nefrologia. Um total de 9 residentes, 4 de Nefrologia e 5 de clínica médica, frequentaram um curso de ultrassom teórico-prático de 16 horas. Foram abordados temas de ultrassom relacionados ao trato urinário, pulmão, coração, vasos sanguíneos, biópsia renal e inserção de cateter venoso central. A avaliação constou de testes cognitivos (TC) de múltipla escolha e associação de imagens antes e após o curso e de avaliação prática de competências na geração de imagens ultrassonográficas e realização de procedimentos pelo Exame Estruturado de Habilidades Clínicas (OSCE). Todos os residentes concluíram o curso. Observou-se melhora significativa dos conhecimentos quando se compararam as notas obtidas pelos residentes antes e após o TC de múltipla escolha e de associação de imagens. Ainda, observou-se que a avaliação de competência dos residentes pelo OSCE, com exceção da estação sobre pulmão, foi considerada muito boa. Concluiu-se que um curso de POCUS em nefrologia de apenas 16 horas, possibilita o desenvolvimento de competências na obtenção de imagens e a realização de procedimentos nefrológicos. O currículo desenvolvido pode servir de modelo para o aprendizado do POCUS em nefrologia, contribuindo assim que para a capacitação do uso da ultrassonografia (US) por não radiologista tem aumentado e decorre da necessidade de o médico integrar "novas" informações clínicas ao processo diagnóstico.

Complementando a discussão, Koratala (2021) realizou um estudo utilizando o POCUS em nefrologia e observou que ainda falta habilidade dos nefrologistas fora do ambiente de pesquisa de utilizar esta tecnologia na sua prática diária. Contudo, há de buscar estratégias para uma maior adesão ao seu uso, visto que:

É bem sabido que a congestão pulmonar está associada a piores desfechos em pacientes com síndromes cardiorrenais. Infelizmente, os achados do exame físico convencional nem sempre são confiáveis na avaliação da volemia. Neste contexto, o POCUS surgiu como um "aprimoramento" atraente para o exame clínico à beira do leito nas duas últimas décadas Koratala (2021, p. 1).

Corroborando com os estudos citados acima, Bastos et al. (2021) em levantamento realizado com sócios da Sociedade Brasileira de Nefrologia observou que "a maioria dos nefrologistas brasileiros entrevistados não foi treinada em ultrassom, contudo, a quase totalidade dos participantes da pesquisa manifestou interesse em aprender a utilizar a POCUS na prática nefrológica".

Phillips et al. (2019) citaram em seu estudo que, à medida que testes mais sensíveis se tornam disponíveis, existe uma preocupação com a contaminação da instrumentação devido a manuseio inadequado, erros cometidos durante o processamento ou contaminação ambiental.

Neste sentido, Soares et al. (2020) afirma que "É necessário capacitar os profissionais no que se refere à segurança das ações concernentes ao cuidado no leito, ao envolvimento do paciente durante todo seu internamento e ao fortalecimento de estratégias fundamentadas nas evidências e na educação permanente da equipe".

Quando se há treinamento eficiente, ocorre um ganho extremo em termos de diagnósticos, é o que aponta estudo de Bastos et al. (2019), em que os estudantes de medicina que focaram seus estudos na tecnologia POCUS, desenvolveram performance diagnóstica superior à de médicos cardiologistas já reconhecidos por sua experiência clínica.

Além do campo da nefrologia, as discussões em torno da necessidade da realização de exames complementares no 
pré-cirúrgico tem sido cada vez maior nos últimos anos.

Podemos atribuir este aumento nas discussões em razão de uma observância e a compreensão dos exageros muitas das vezes realizados em torno da exigência de exames que somente oneram os custos hospitalares, atrasam a realização dos procedimentos, aumentam a demanda por serviços e ainda podem ter interferência sobre a segurança do paciente.

Assim sendo, o que se observa é, na verdade, um processo de racionalização dos exames, em uma tendência mundial observada na atualidade, conforme os estudos aqui selecionados puderam comprovar.

Muitos dos exames até bem pouco tempo atrás exigidos em um pré-operatório, hoje já se observa que, na realidade, não são indispensáveis, e poderão ser evitados, salvo em casos clínicos específicos.

Observou-se também o consenso de que, via de regra, a avaliação clínica, através de uma anamnese detalhada, é o essencial e possibilita que seja oferecido ao paciente o menor risco cirúrgico possível.

Corroborando com esta visão, Guerra et al. (2012) considera que um exame clínico bem detalhado é essencial e indispensável e também o suficiente para que haja uma diminuição de até $60 \%$ a quantidade de exames pré-operatórios.

Mesma visão foi defendida por Leal, Silva e Oliveira (2013) e Giordano, Giordano e Giordano (2009) que também concordaram que a avaliação pré-operatória adequada é fundamental para otimizar o respectivo procedimento para cada paciente, reduzindo riscos e custos.

Dentro deste contexto é que estão inseridos os testes POC, considerados na prática como uma importante ferramenta para o diagnóstico mais rápido e preciso de uma infinidade de doenças e condições clínicas que, na maioria das vezes, requerem uma intervenção precoce e rápida.

\section{Considerações Finais}

Através do levantamento bibliográfico realizado, é possível concluir que a tecnologia POC é sobremodo importante, até mesmo essencial para que se possa determinar o diagnóstico definitivo e confirmatório de uma série de condições patológicas e clínicas no cotidiano do atendimento hospitalar, especialmente. Esta tecnologia deve ser cada vez mais estimulada na prática, tendo em vista não somente o fato de que permite um diagnóstico precoce, mas também porque possibilita um melhor prognóstico para aqueles casos considerados mais graves, contribuindo para que vidas sejam salvas.

Embora os desfechos a longo prazo da terapia guiada por POC tenham que ser estabelecidos através de ensaios clínicos maiores, constitui-se em um grande acréscimo ao conjunto de habilidades a serem estrategicamente implementadas nos diversos serviços e ramos da saúde.

A nosso entendimento, cada vez mais a tecnologia e o preparo de profissionais para o uso correto destas ferramentas deve ser estimulado e realizado, garantindo assim um melhor prognóstico aos pacientes a partir de um diagnóstico preciso, rápido e ainda com menores custos aos hospitais e aos pacientes, vantagens estas que a tecnologia POC proporciona e que se constituem em seu grande diferencial.

No entanto, vale ressaltar que existem lacunas na literatura sobre a utilização da tecnologia POC, pois ainda é algo novo para muitos e até mesmo subutilizado, podendo ser expandido para muitas áreas da saúde e regiões com déficits importantes no que tange aos recursos humanos e materiais. Sendo esta uma fonte de futuras pesquisas na área.

\section{Referências}

Aljadi, Z., Kalm, F., Ramachandraiah, H., Nopp, A., Lundahl, J., \& Russom, A. (2019). Microfluidic immunoaffinity basophil activation test for point of care allergy diagnosis. Journal of Applied Laboratory Medicine, 4 (2), 152-163. https://pubmed.ncbi.nlm.nih.gov/31639660/.

Arienzo, A., Cellitti, V., Ferrante, V., Losito, F., Stalio, O., Murgia, L., Marino, R., Cristofano, F., Orrú, M., Visca, P., Di Somma, S., Silvestri, L., Ziparo, V., \& Antonini, G. (2019). A new point-of-care test for the rapid detection of urinary tract infections. European Journal of Clinical Microbiology \& Infectious 
Diseases, $\quad 39, \quad 325-332 . \quad$ https://pubmed.ncbi.nlm.nih.gov/31707506/\#: :text=The\%20Micro\%20Biological\%20Survey\%20\%28MB S\%29\%20POCT\%20is\%20a,the\%20potentials\%20and\%20limits\%20of\%20the\%20MBS\%20POCT.

Barcellona, D., Fenu, L., \& Marongiu, F. (2017). Point-of-care testing INR: an overview. Clinical Chemistry and Laboratory Medicine, 55 (6), 800-805. https://pubmed.ncbi.nlm.nih.gov/27754958/.

Bastos, M. G., Ronzani, F. A. T., Carmo, W. B., Toledo, G. C., \& Paula, R. B. (2019). Integração do ensino da ultrassonografia point of care no currículo de graduação em medicina: um relato de experiência. Hospital Universitário Revista, 45 (1), 98-103. https://periodicos.ufjf.br/index.php/hurevista/article/view/13977/18907.

Bastos, M. G.L., Nascimento, M. M., Barros, E., Pazeli, J. M., \& Kirsztajn, G. M. (2021). Ultrassonografia point-of-care em nefrologia: uma pesquisa nacional transversal entre nefrologistas brasileiros. $\quad$ Brazilian 43 Journal https://www.scielo.br/j/jbn/a/d6SBpmD5GXHPTsn3bHS43fB/?format=pdf\&lang=pt.

Brasil. (2021). Ministério da Saúde. Secretaria de Ciência, Tecnologia, Inovação e Insumos Estratégicos em Saúde. Teste diagnóstico, point of care, de Cryptococcal Antigen Lateral Flow Assay (CRAG-LFA) para detecção de infecção por Cryptococcus e diagnóstico de meningite criptocócica em pessoas vivendo com o vírus da imunodeficiência humana (PVHIV). https://pesquisa.bvsalud.org/portal/resource/pt/biblio-1253427.

Bollinger, D., \& Tanaka, K. A. (2017). Point of care coagulation testing in cardiac surgery. Seminars Thrombosis and Hemostasis, 43 (4), 386-396. https://pubmed.ncbi.nlm.nih.gov/28359133/.

Celer Biotecnologia. (2021). Tecnologias Point of Care (POC) em processo acelerado de inovação. https://celer.ind.br/tecnologias-point-of-care-poc-emprocesso-acelerado-de-inovacao/.

Crocker, J.B., Lee-Lewandrowski, E., Lewandrowski, N., Baron, J., Gregory, K., \& Lewandrowski, K. (2014) Implementation of Point-of-Care Testing in an Ambulatory Practice of an Academic Medical Center. American Journal of Clinical Pathology,142, 640-646. https://pubmed.ncbi.nlm.nih.gov/25319979/.

Dusse, L. M. S., Oliveira, N. C., Rios, D. R. A., \& Marcolino, M. S. (2012) RNI Point of care test (POCT): esperança ou ilusão? Brazilian Journal of Cardiovascascular Surgery, 27 296-301. https://www.scielo.br/j/rbccv/a/rQyjdVLPGVgQpL7HjxHp3Sh/\#: :text=Na\%20\%C3\%BAltima\%20d\%C3\%A9cada\%2C\%20foram\%20desenvolvidos\%20os $\% 20$ testes\%20point-of-care,\%28RNI-POCT\%29\%2C\%20constitui\%20o\%20principal\%20xame\%20dessa\%20nova\%20proposta.

Giordano, L. A., Giordano, M. V., \& Giordano, R. O. S. (2009). Exames pré-operatórios nas cirurgias ginecológicas eletivas. Femina, 37 (11). http://bases.bireme.br/cgi-

bin/wxislind.exe/iah/online/?IsisScript=iah/iah.xis\&base=LILACS\&lang=p\&nextAction=lnk\&exprSearch=545661\&indexSearch=ID.

Guerra, M. E., Pereira, C. S., Falcão, D. P., Sigwalt, M. F., \& Skinovsky, J. (2012). Análise da relevância dos exames laboratoriais pré-operatórios solicitados em cirurgias eletivas em um hospital universitário. Revista do Médico Residente, 14 (1), 47-53. http://crmpr.org.br/publicacoes/cientificas/index.php/revistado-medico-residente/article/download/145/144.

Koratala, A. (2021). A utilização de ultrassom point-of-care na clínica cardiorrenal para melhorar o atendimento ao paciente. Brazilian Journal Nephrology,43 (1), 136-137. https://www.scielo.br/j/jbn/a/rhZPTKTmGK5jMxJ4DwtBvNG/?lang=en.

Leal, F. P., Silva, A. P., \& Oliveira, E. S. (2013). Avaliação pré-operatória: exames complementares de rotina? Brazilian Journal of Surgery and Clinical Research, 4 (1), 49-55. https://www.recantodasletras.com.br/artigos-de-saude/4423928

Lusignan, S., Hoang, U., Liyanage, H., Yonova, I., Ferreira, F., Diez-Domingo, J., \& Clark, T. (2019). Feasibility of point-of-care testing for influenza withing a national primary care sentinel surveillance network in england: protocol for a mixed methods study. JMIR Research Protocols, 8 (11), e14186. https://www.google.com.br/url?sa=t\&rct=j\&q=\&esrc=s\&source=web\&cd=\&cad=rja\&uact=8\&ved=2ahUKEwjBx5Ta4c_0AhW4IrkGHUhQAnUQFnoECAU QAQ\&url=https\%3A\%2F\%2Fpubmed.ncbi.nlm.nih.gov\%2F31710303\%2F\&usg=AOvVaw2UCKJ9RzLfJ7TF49YWvHiT.

Marconi, M. A. \& Lakatos, E. M. (2017). Fundamentos de metodologia científica. (8a ed). Atlas.

Noronha, R. T. H. (2019). Point-of-care na região autónoma dos açores. Caracterização sociodemográfica da utilização por profissionais de saúde. Perspectivas futuras no âmbito da telemedicina (MHEALTH). Dissertação de Mestrado. Universidade de Lisboa, Lisboa, Portugal. https://repositorio.ul.pt/bitstream/10451/43177/1/TM_Ricardo_Homem_Noronha.pdf

Nunes, A. A., Pazeli Junior, J. M., Rodrigues, A. T., Tollendal, A. L. S. V., Ezequiel, O. S., Colugnati, F. A. B., \& Bastos, M. G. (2016). Desenvolvimento de competências para o uso da ultrassonografia point-of-care em Nefrologia. Brazilian Journal of Nefhrology, 38 (2), 209-214. https://www.google.com.br/url?sa=t\&rct=j\&q=\&esrc=s\&source=web\&cd=\&cad=rja\&uact=8\&ved=2ahUKEwjFur2o4s_0AhWlH7kGHb6PBtYQFnoECAUQ AQ\&url=https $\% 3 \mathrm{~A} \% 2 \mathrm{~F} \% 2 \mathrm{Fwww}$. bjnephrology.org $\% 2$ Farticle $\% 2$ Fdesenvolvimento-de-competencias-para-o-uso-da-ultrassonografia-point-of-care-emnefrologia\%2F\&usg=AOvVaw3mICd5q6ovEQrCL_yraq1n.

Phillips, J.., Mccune, S., Fantz, C. R., Engstrom-Melnyk, J., \& Osiecki, J. C. (2019). Assay integrity of a PCR influenza point of care test remains following artificial system contamination. Journal of Applied 4 Laboratory $422-426$. https://www.google.com.br/url?sa=t\&rct=j\&q=\&esrc=s\&source=web\&cd=\&cad=rja\&uact=8\&ved=2ahUKEwimlsGE48_0AhUsF7kGHQGgDccQFnoECAM QAQ\&url=https\%3A\%2F\%2Fpubmed.ncbi.nlm.nih.gov\%2F31659080\%2F\&usg=AOvVaw2MirxrXIwuQ2bEBsefGse8.

Schiling, U. M. (2014). Time is Money - the Economic Impact of Point of Care on the Emergency Department of a Tertiary Care University Hospital. Point of Care, 13, 21-23.https://journals.lww.com/poctjournal/Abstract/2014/03000/Time_is_Money_the_Economic_Impact_of_Point_of_Care.6.aspx.

Soares, D. T. S., Hermann, A. P., Lacerda, M. R., Méier, M.J., Cáceres, N. T. G., \& Lima, J. Z. (2020). Cuidados ao paciente crítico na realização do exame de imagem no leito: revisão integrativa. Revista Brasileira de Enfermagem, 73 (6), e20180948. https://www.scielo.br/j/reben/a/QvZKQwZ3r9nV5PNLWyg8FbN/?format=pdf\&lang=pt 